\title{
Global metabolomic and lipidomic analysis reveals the potential mechanisms of hemolysis effect of Ophiopogonin D and Ophiopogonin D' in vivo
}

Huan-Hua $\mathrm{Xu}^{1,2+}$, Zhen-Hong Jiang ${ }^{3 \dagger}{ }$, Cong-Shu Huang ${ }^{2,4}$, Yu-Ting Sun ${ }^{2}$, Long-Long Xu ${ }^{2,5}$, Xiang-Ling Tang ${ }^{2}$, Hong-Ling Tan², Zeng-Chun $\mathrm{Ma}^{2}$ and Yue Gao ${ }^{1,2^{*}}$

\begin{abstract}
Background: OPD and OPD' are the two main active components of Ophiopogon japonicas in Shenmai injection (SMI). Being isomers of each other, they are supposed to have similar pharmacological activities, but the actual situation is complicated. The difference of hemolytic behavior between OPD and OPD'in vivo and in vitro was discovered and reported by our group for the first time. In vitro, only OPD' showed hemolysis reaction, while in vivo, both OPD and OPD' caused hemolysis. In vitro, the primary cause of hemolysis has been confirmed to be related to the difference between physical and chemical properties of OPD and OPD'. In vivo, although there is a possible explanation for this phenomenon, the one is that OPD is bio-transformed into OPD' or its analogues in vivo, the other one is that both OPD and OPD' were metabolized into more activated forms for hemolysis. However, the mechanism of hemolysis in vivo is still unclear, especially the existing literature are still difficult to explain why OPD shows the inconsistent hemolysis behavior in vivo and in vitro. Therefore, the study of hemolysis of OPD and OPD' in vivo is of great practical significance in response to the increase of adverse events of SMI.
\end{abstract}

Methods: Aiming at the hemolysis in vivo, this manuscript adopted untargeted metabolomics and lipidomics technology to preliminarily explore the changes of plasma metabolites and lipids of OPD- and OPD'-treated rats. Metabolomics and lipidomics analyses were performed on ultra-high performance liquid chromatography (UPLC) system tandem with different mass spectrometers (MS) and different columns respectively. Multivariate statistical approaches such as principal component analysis (PCA) and orthogonal partial least square-discriminant analysis (OPLS-DA) were applied to screen the differential metabolites and lipids.

Results: Both OPD and OPD' groups experienced hemolysis, Changes in endogenous differential metabolites and differential lipids, enrichment of differential metabolic pathways, and correlation analysis of differential metabolites and lipids all indicated that the causes of hemolysis by OPD and OPD' were closely related to the interference of phospholipid metabolism.

\footnotetext{
*Correspondence: gaoyue@bmi.ac.cn

${ }^{\dagger}$ Huan-Hua Xu and Zhen-Hong Jiang contributed equally to this work

${ }^{1}$ Tianjin University of Traditional Chinese Medicine, Tianjin 300193, China

Full list of author information is available at the end of the article
}

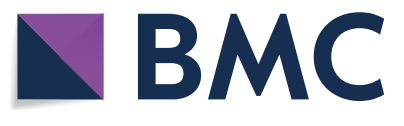

(c) The Author(s) 2021. This article is licensed under a Creative Commons Attribution 4.0 International License, which permits use, sharing, adaptation, distribution and reproduction in any medium or format, as long as you give appropriate credit to the original author(s) and the source, provide a link to the Creative Commons licence, and indicate if changes were made. The images or other third party material in this article are included in the article's Creative Commons licence, unless indicated otherwise in a credit line to the material. If material is not included in the article's Creative Commons licence and your intended use is not permitted by statutory regulation or exceeds the permitted use, you will need to obtain permission directly from the copyright holder. To view a copy of this licence, visit http://creativeco mmons.org/licenses/by/4.0/. The Creative Commons Public Domain Dedication waiver (http://creativecommons.org/publicdomain/ zero/1.0/) applies to the data made available in this article, unless otherwise stated in a credit line to the data. 
Conclusions: This study provided a comprehensive description of metabolomics and lipidomics changes between OPD- and OPD'-treated rats, it would add to the knowledge base of the field, which also provided scientific guidance for the subsequent mechanism research. However, the underlying mechanism require further research.

Keywords: Hemolysis, Ophiopogonin D, Ophiopogonin D', Metabolomic, Lipidomic

\section{Background}

Shenmai injection (SMI) is a widely used traditional Chinese medicine (TCM) extracted from Panax ginseng and Ophiopogon japonicas [1]. It has been widely used to treat different disease for its excellent therapeutic effects, such as the shock of qi and yin deficiency $[2,3]$, coronary heart disease $[4,5]$, viral myocarditis [6], chronic pulmonary heart disease [7], and neutropenia [8]. It can also strengthen the immune system of cancer patients to help prevent cancer $[9,10]$. When used in combination with chemotherapy drugs, it has a certain synergistic effect and can reduce the side effects caused by chemotherapy drugs [11]. In the pneumonia pandemic caused by the new coronavirus (COVID-19) in early 2020 in China [12], SMI was listed as a recommended medication due to its positive effect on the improvement of patients' blood oxygen saturation by the Novel Coronavirus Pneumonia Diagnosis and Treatment Plan (Provisional 7th Edition), which was released by the Chinese government [13].

Ophiopogonin D (OPD) and Ophiopogonin D' (OPD') are two main active components in Ophiopogon japonicas with the same molecular formula and similar structure, only show the different substitution of sugar side chains (Fig. 1). Previous studies on active components of Ophiopogon japonicas always focused on OPD, and have found that OPD was an effective ingredient for the treatment of cardiovascular and cerebrovascular diseases [14, 15]. OPD has antioxidant activity and can promote cell proliferation, stabilize mitochondrial membrane potential, reduce calcium influx, inhibit angiotensin II as well as induce liver microsomal enzymes [16]. However, since 2017, there have been reports of cytotoxicity of OPD' at the cellular level. Lu's et.al found that OPD' could significantly inhibit the growth of androgen-independent prostate cancer PC3 cells, and was related to the RIP1/MLKL pathway [17, 18]. When H9c2 [19] cardiomyocytes and HepG2 [20] human hepatoma cells were treated with OPD' $\left(>5 \mu \mathrm{mol} \cdot \mathrm{L}^{-1}\right)$ for $24 \mathrm{~h}$, cells were suffered from cell morphology shrank, the number of nuclei decreased, ROS content increased, mitochondrial membrane potential decreased and the rate of apoptosis increased. The survival rate of cells gradually decreased with the extension of the treatment time of OPD', and the toxic reaction became more and more obvious.

In our previous work, we discovered for the first time that the hemolytic behavior of OPD' is completely different from OPD (unpublished manuscript). Despite SMI was widely used in clinical and has gained a good reputation, the risk assessment of SMI based on cytotoxicity and hemolytic of OPD' is still missing, limiting our ability to improve the safety of SMI. Also, with the widespread clinical application, the adverse reaction events of SMI are increasing. From January 2012 to December 2014, only the Hubei Provincial Adverse Drug Reaction Testing Center collected more than 1,500 cases of adverse reactions reports of SMI [21]. Existing adverse event analysis for the collected SMI indicates that there was a great correlation between adverse events and hemolysis. But the mechanism of hemolysis in vivo is still vague.

Here, we further specific manifestations in hemolytic behavior between OPD and OPD' in vivo, and untargeted metabolomics and lipidomics were adopted to study the underlying mechanisms. In the in vivo hemolysis test, both OPD and OPD' affected the content of hemoglobin (HGB), red blood cell (RBC) count and reticulocyte (RET) count in the blood. We also first identified different metabolites by pairwise comparison of different groups,

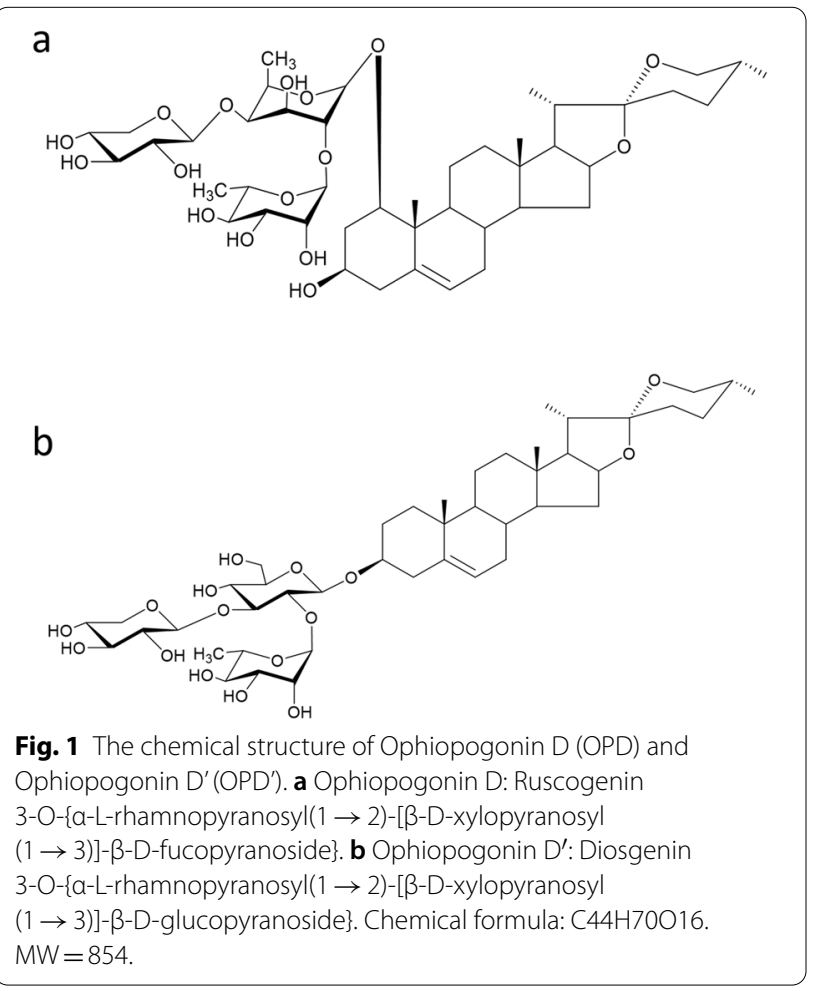


and then further classified the differential metabolites by function. We found that both OPD and OPD' could induce hemolysis in vivo by interfering with phospholipid metabolism, promoting the production of lysophospholipids (LysoPC), and causing damage to the red blood cell membrane. Although the direct cause of hemolysis is inseparable from the disorder of phospholipid metabolism, however, how OPD and OPD' participated in this process needs further exploration.

\section{Materials and methods}

\section{Chemicals and reagents}

OPD (F05975, purity: 98\% by HPLC) and OPD' (F581298, purity: $98 \%$ by HPLC) were purchased from Shanghai EFE Biotechnology Co., Ltd. (Shanghai, China). Qualitative detection kit for urine hemosiderin test (Rous) was purchased from Shanghai Yuan Mu Biotechnology Co., Ltd. (Shanghai, China).

All other chemicals and solvents were analytical or HPLC grade. The ultra-pure water was prepared by the Milli-Q system (Millipore, Bedford, MA, USA). Methanol, acetonitrile, formic acid were purchased from $\mathrm{CNW}$ Technologies. GmbH (Düsseldorf, Germany). L-2-chlorophenylalanine was purchased from Shanghai Heng Chuang Bio-technology Co., Ltd. (Shanghai, China).

\section{Animals}

In vivo hemolytic study was conducted in the apparently healthy adult Wistar rats (180-200 g, male and female). Animals were purchased from Beijing Charles River Animal Breeding Center (Production license: SCXK (Jing)-2018-0010). All animals were housed in an environmentally controlled breeding room (temperature: $22 \pm 2{ }^{\circ} \mathrm{C}$, humidity: $50 \pm 5 \%$, dark/light cycle: $12 / 12 \mathrm{~h}$ ). The animals were provided standard laboratory food and water. The experimental protocols were approved by the Animal Ethics Committee of Academy of Military Medical Sciences (No. IACUC-DWZX-2020-684) and were performed in accordance with the guidelines of the National Institutes of Health for the Care and Use of Laboratory Animals.

\section{Parameters related to hemolysis}

In this experiment, the groups were set as follows: normal control group (NC), OPD independent use group (OPD), OPD' independent use group (OPD'), OPD and OPD' combination group (OPD + OPD'), OPD and OPD' interval administration group (OPD $\rightarrow$ OPD'). 25 Wistar rats ( 5 rats in each group) were given by tail vein injection once a day for 30 days. The activities and physiological conditions of rats were observed and recorded every day, body weight were weighed every 7 days. At the end of experiments, carbon dioxide anesthesia, whole blood was collected, anticoagulation and perform hematology analysis. Plasma was separated by centrifugation for biochemical analysis. Urine were collected, one part was used to determine whether it contains hemosiderin by Rous method, another part was used for routine urine testing.

\section{Metabolomic experiments Sample preparation}

22 rats were randomly assigned to 3 groups, they are normal control group $(\mathrm{NC}, \mathrm{n}=6)$, OPD group $(\mathrm{n}=8)$ and OPD' group $(\mathrm{n}=8)$. All drugs were administrated by intravascular injection for 14 days. According to the limit of clinical hemolysis rate $(5 \%)$ and the results obtained from in vitro hemolytic test, When the concentration of OPD' exceeds $5 \mu \mathrm{g} \cdot \mathrm{ml}^{-1}$, the hemolysis rate exceeds the clinical limit of $5 \%$. Assuming an ideal situation, according to the blood volume of the experimental animals and this blood drug concentration (i.e. $5 \mu \mathrm{g} \cdot \mathrm{ml}^{-1}$ ), the doses of the OPD and OPD' of each group are defined as $0.25 \mathrm{mg} \cdot \mathrm{kg}^{-1}$. Plasma was prepared by centrifugation for metabolomics analysis.

$100 \mu \mathrm{L}$ of plasma was added to a $1.5 \mathrm{~mL}$ Eppendorf tube with $10 \mu \mathrm{L}$ of 2 -chloro-1-phenylalanine $\left(0.3 \mathrm{mg} \cdot \mathrm{mL}^{-1}\right)$ dissolved in methanol as internal standard. Subsequently, $300 \mu \mathrm{L}$ of ice-cold mixture of methanol and acetonitrile $(2 / 1, \mathrm{v} / \mathrm{v})$ was added, and the mixtures were vortexed for $1 \mathrm{~min}$, ultrasonicated at ambient temperature (25$28{ }^{\circ} \mathrm{C}$ ) for $10 \mathrm{~min}$, store at $-20{ }^{\circ} \mathrm{C}$ for $30 \mathrm{~min}$. The extract was centrifuged at $13,000 \mathrm{rpm}, 4{ }^{\circ} \mathrm{C}$ for $15 \mathrm{~min}, 300 \mu \mathrm{L}$ of supernatant in a brown and glass vial was dried in a freeze concentration centrifugal dryer, $400 \mu \mathrm{L}$ mixture of methanol and water $(1 / 4, \mathrm{v} / \mathrm{v})$ were added to each sample and then vortexed for $30 \mathrm{~s}$, Repeat the centrifugation operation, $150 \mu \mathrm{L}$ supernatants from each tube were collected using crystal syringes, filtered through $0.22 \mu \mathrm{m}$ microfilters and transferred to LC vials for LC-MS analysis. QC samples were prepared by mixing aliquots of the all to be a pooled sample and the QCs were injected at regular intervals (every 10 samples) throughout the analytical run to provide a set of data from which repeatability can be assessed.

\section{UPLC-MS/MS analysis}

Instrument for this study is a LC-MS system composed of Dionex U3000 UPLC ultra-efficient liquid chromatography tandem a QE plus high-resolution mass spectrometer. Liquid phase separation was performed on an Acquity UPLC HSS T3 $(100 \mathrm{~mm} \times 2.1 \mathrm{~mm}, 1.8 \mu \mathrm{m})$ column. The flow rate was controlled at $0.35 \mathrm{~mL} \cdot \mathrm{min}^{-1}$, the temperature of the automatic injector was maintained 
at $4{ }^{\circ} \mathrm{C}$, and the column temperature was controlled at $50{ }^{\circ} \mathrm{C}$. The injection volume of all samples was $5 \mu \mathrm{L}$. The mobile phase consisted of water and acetonitrile which both containing $0.1 \%$ formic acid. Gradient elution was performed as follows: (1) mobile phase A was at $95 \%$ at $0 \mathrm{~min},(2)$ an isocratic elution was maintained at $95 \% \mathrm{~A}$ from 0 to $1 \mathrm{~min}$, (3) a linear gradient was decreased to $0 \%$ A from 1 to $11 \mathrm{~min}$, and (4) mobile phase A maintained at $0 \%$ from 11 to $13 \mathrm{~min}$.

The condition of mass spectrometry (MS) analysis was ESI source ionization in positive and negative ion mode respectively, and the scanning mode adopted centroid and continuum mode. The optimum conditions were shown in Table 1.

\section{Lipidomic experiments Sample preparation}

After the same steps of metabolomic sample preparation. $100 \mu \mathrm{L}$ of plasma was added to a tube with $20 \mu \mathrm{L}$ of 2-chloro-1-phenylalanine $\left(0.3 \mathrm{mg} \cdot \mathrm{mL}^{-1}\right)$ and Lyso PC $(17: 0)\left(0.01 \mathrm{mg} \cdot \mathrm{mL}^{-1}\right)$ dissolved in methanol as internal standard. Subsequently, $300 \mu \mathrm{L}$ Isopropanol was added, and vortex for $30 \mathrm{~s}$, and extract with ultrasound for $10 \mathrm{~min}$. Place at $-20{ }^{\circ} \mathrm{C}$ for $30 \mathrm{~min}$, centrifuge for $10 \mathrm{~min}$, take $300 \mu \mathrm{L}$ of supernatant and transfer to a new tube. Another $200 \mu \mathrm{L}$ of isopropanol was added to the original tube and extract again, take $200 \mu \mathrm{L}$ up layer and merge it into the new tube. After drying, the lipid residue in the centrifuge tube was reconstituted with $200 \mu \mathrm{L}$ isopropanol-methanol mixture $(1: 1, \mathrm{~V} / \mathrm{V})$, vortex $30 \mathrm{~s}$, ultrasound $3 \mathrm{~min}$, centrifuged for $10 \mathrm{~min}\left(12000 \mathrm{rpm}, 4{ }^{\circ} \mathrm{C}\right)$, take $150 \mu \mathrm{L}$ of the supernatant and put it into an LC-MS sample vial for LC-MS analysis. QC samples were prepared by mixing equal volumes of extracts from all samples, and each QC has the same volume as the sample.

Table 1 The condition of mass spectrometry (MS) analysis

\begin{tabular}{lll}
\hline Parameter & ESI positive & ESI negative \\
\hline Spray Voltage $(\mathrm{V})$ & 3800 & 3000 \\
Capillary Temperature $\left({ }^{\circ} \mathrm{C}\right)$ & 320 & 320 \\
Aux gas heater temperature $\left({ }^{\circ} \mathrm{C}\right)$ & 350 & 350 \\
Sheath Gas Flow Rate $($ Arb) & 35 & 35 \\
Aux gas flow rate (Arb) & 8 & 8 \\
S-lens RF level & 50 & 50 \\
Mass range (m/z) & $70-1000$ & $70-1000$ \\
Full ms resolution & 70,000 & 70,000 \\
MS/MS resolution & 17,500 & 17,500 \\
NCE/stepped NCE & 20,40 & 20,40 \\
\hline
\end{tabular}

\section{UPLC-MS/MS analysis}

The lipidomic analyses were performed on Q Exactive Mass Spectrometer (Thermo Fisher Scientific Inc., Waltham, MA, USA) coupled to Nexera UPLC system (Shimadzu, Kyoto, Japan). An ACQUITY UPLC BEH C18 $(2.1 \mathrm{~mm} \times 100 \mathrm{~mm}, 1.7 \mu \mathrm{m}$, Waters, Milford, MA, USA) was used for separation of lipids. The mobile phase for HPLC was composed of solvent A $(10 \mathrm{mM}$ ammonium formate and $0.1 \%$ formic acid in acetonitrile/water $(60: 40))$ and solvent B (10 mM ammonium formate and $0.1 \%$ formic acid in isopropanol/ acetonitrile (90:10)). LC gradient was as follows: $0.0-3.0 \mathrm{~min}$ for $30 \% \mathrm{~B}, 3.0-$ $5.0 \mathrm{~min}$ to $62 \% \mathrm{~B}, 5.0-15.0 \mathrm{~min}$ to $82 \% \mathrm{~B}, 15.0-16.5 \mathrm{~min}$ to $99 \% \mathrm{~B}, 16.5-18.0 \mathrm{~min}$ to maintain $99 \% \mathrm{~B}$. The flow rate was $0.35 \mathrm{~mL} / \mathrm{min}$, and the injected sample amount was $5 \mu \mathrm{L}$. Heated electrospray ionization (HESI) positive and negative ion modes were used for detection. Positive: Heater Temp $300{ }^{\circ} \mathrm{C}$, Sheath Gas Flow rate 45 arb, Aux Gas Flow Rate15 arb, Sweep Gas Flow Rate 1arb, spray voltage $3.5 \mathrm{KV}$, Capillary Temp $320{ }^{\circ} \mathrm{C}$, S-Lens RF Level 50\%. MS1 scan ranges: $120-1800$. Negative: Heater Temp $300{ }^{\circ} \mathrm{C}$, Sheath Gas Flow rate 45arb, Aux Gas Flow Rate $15 \mathrm{arb}$, Sweep Gas Flow Rate 1arb, spray voltage $3.1 \mathrm{KV}$, Capillary Temp $320^{\circ} \mathrm{C}$, S-Lens RF Level 50\%. MS1 scan ranges: $120-1800$.

\section{Data processing and statistical analysis}

The acquired LC-MS raw data were analyzed by the progqenesis QI software (Waters Corporation, Milford, USA). The Excel file was obtained with three dimensions datasets including $\mathrm{gm} / \mathrm{z}$, peak RT and peak intensities, and $\mathrm{RT}-\mathrm{m} / \mathrm{z}$ pairs were used as the identifier for each ion. The resulting matrix was further reduced by removing any peaks with missing value (ion intensity $=0$ ) in more than $50 \%$ samples.

Metabolites were identified by progenesis QI (Waters Corporation, Milford, USA) Data Processing Software, based on public databases such as http://www.hmdb. ca/; http://www.lipidmaps.org/ and self-built databases. The software Lipid search (Thermo Fisher Scientific Inc., Waltham, MA, USA) was employed for peak picking and alignment of lipids.

The positive and negative data were combined to get a combine data which was imported into $\mathrm{R}$ package. Principle component analysis (PCA) and (orthogonal) partial least-squares-discriminant analysis (OPLS-DA) were carried out to visualize the metabolic alterations among experimental groups, after mean centering and Pareto variance scaling, respectively. The Hotelling's T2 region, shown as an ellipse in score plots of the models, defines the 95\% confidence interval of the modeled variation. Variable importance in the projection (VIP) ranks 
the overall contribution of each variable to the OPLS-DA model, and those variables with VIP $>1$ are considered relevant for group discrimination.

\section{Results}

The hemolytic behavior of OPD and OPD' in vivo

As it was shown in Fig. 2 and Table 2, Hemolysis was found in each drug treatment group, and hematological indicators related to hemolysis, such as RBC, HGB, RET, and RET\%, were significantly changed. Compared with the NC group, RBC and HGB decreased significantly, while RET and RET\% were significantly increased, which means that RBCs were destroyed faster, and the immature RBCs (i.e. RET) were released to the peripheral blood increased. Indicated that the lifespan of RBC may be shortened. The results of the urine examination showed that the urobilinogen (URO) of each treatment group changed significantly, and compared with the NC group, they were all positive
Table 2 Parameters related to hemolysis in each group by urine examination

\begin{tabular}{llll}
\hline Group & Hemosiderin & RBCs in urine & Urobilinogen \\
\hline $\mathrm{NC}$ & - & - & - \\
$\mathrm{OPD}$ & + & + & + \\
OPD' & ++ & + & ++ \\
OPD + OPD' & ++ & ++ & ++ \\
OPD $\rightarrow$ OPD' & ++ & ++ & ++ \\
\hline
\end{tabular}

(degree $1+$ to $2+$. Under normal circumstances, there should be no red blood cells, urobilinogen and hemosiderin in the urine, which is represented by "-". However, with the occurrence and aggravation of hemolysis, the number and concentration of RBC, URO, and hemosiderin will increase, expressed on a scale from $1+$ to $4+$.$) . The results of the urine occult blood tests were$

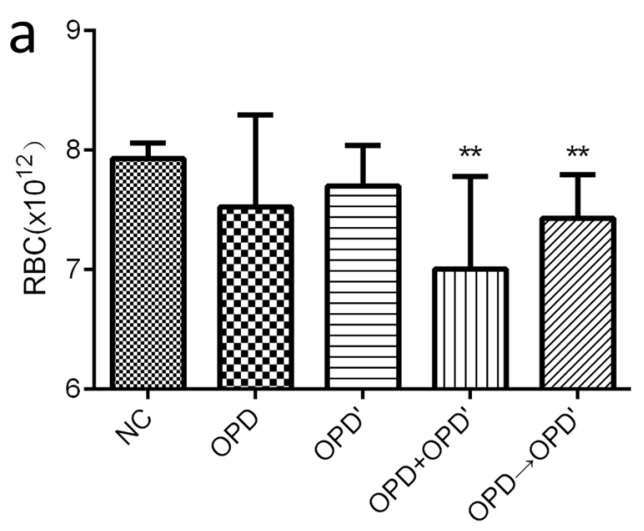

Group

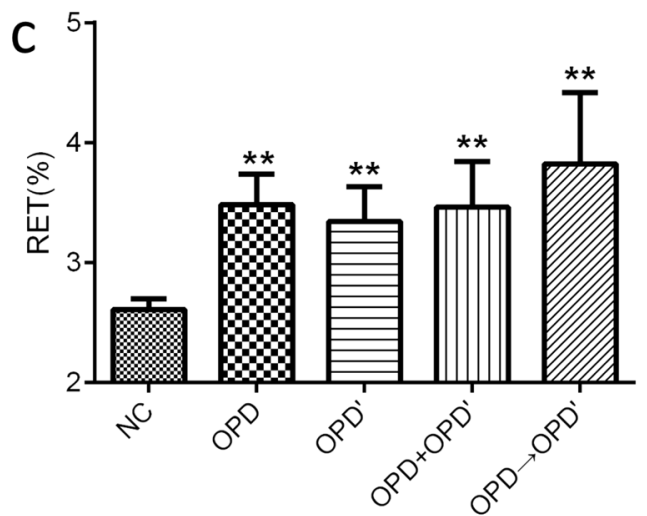

Group

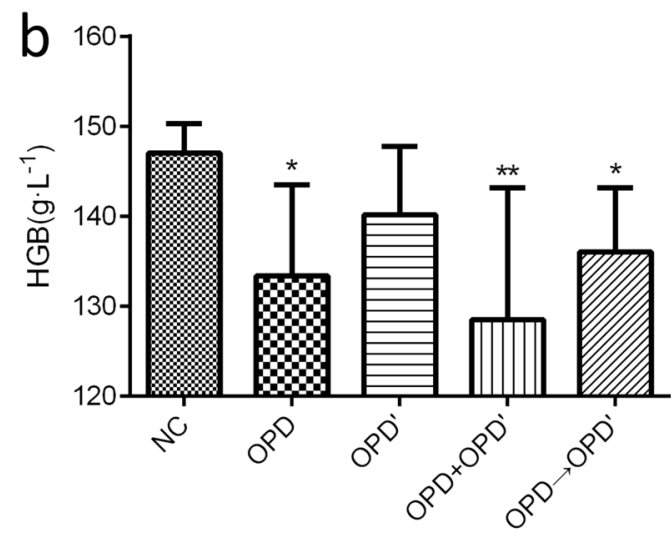

Group

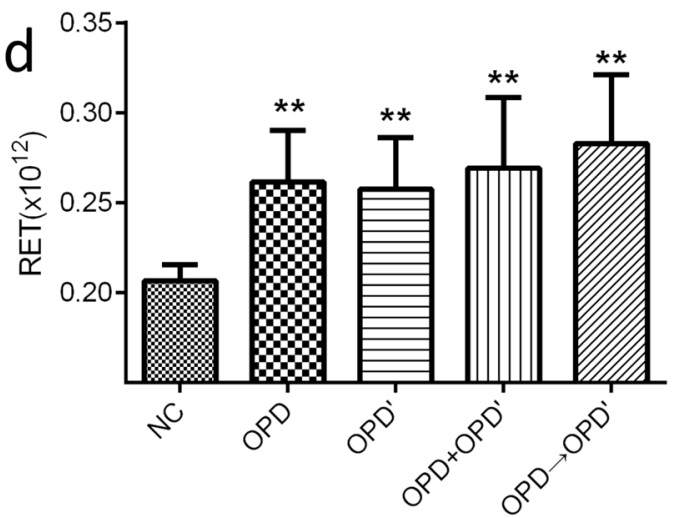

Group

Fig. 2 Parameters related to hemolysis in each group by hematology analysis. a RBC count. b HGB content. c RET count. d RET\%. The error bars represent the standard deviation of measurements for 5 parallel samples in five separate groups $(n=5)$. " "* means that compared with the NC group, the statistical was significant $(p<0.05)$, ${ }^{\prime * * \prime \prime}$ means that compared with the NC group, the statistical was very significant $(p<0.01)$ 
consistent with the results of the URO test. The Rous method [22] was used to determine the hemosiderin in urine, which is a commonly used clinical test to diagnose intravascular hemolysis. As shown in Table 2, obviously scattered or piles of blue glitter particles were observed in each treatment group (degree $1+$ to $2+)$. These indicators confirm that OPD and OPD' can cause intravascular hemolysis [23] when used alone or in combination.

\section{Global metabolic and lipidomic shifts induced by OPD and OPD'}

The metabolic and lipidomic profiles were acquired under positive and negative ionization modes using UPLC-MS. A total of 10,364 ions in ESI $(+)$ and 9741 ions in ESI (-) were obtained by non-targeted metabolic profiling. In lipidomic profiling, 313 ions in ESI $(+)$ and 260 ions in ESI (-) were obtained. PLS-DA score plots were applied to identify the differential metabolites or lipids among OPD and OPD' groups. Figure 3 showed the clear segregation of OPD and OPD' from NC group, indicating that both OPD and OPD' induced obvious disturbance of inter-cellular metabolites and lipids.

\section{Metabolic changes induced by OPD and OPD' in vivo}

According to OPLS-DA model, Variable importance of projection (VIP) value was obtained, and VIP $>1$ is the one of criterion for screening potential biomarkers. In each comparison group, the number of differential metabolites we found were 145 (NC vs OPD), 124 (NC vs OPD') and 84 (OPD vs OPD'), respectively. The volcano graph can be used to visualize the $\mathrm{p}$ value and Fold change value, which is helpful for screening different metabolites (Additional file 1: Figures S1 and S2). Top 20 differential metabolites were listed in Table 3 among different comparison group, and the distribution of fold changes of metabolites in different comparison group from the perspective of sub-class were shown in Fig. 4.

As it were shown in Table 3 and Fig. 4. Compared with the NC group, OPD and OPD' group had similar results in terms of the type, quantity and VIP value of the differential metabolites. In top 20 differential metabolites, LysoPC account for 45\% in OPD and OPD' group, while the phospholipid differential metabolites account for $75 \%$. The abnormal increase of LysoPC and the disorder of phospholipid metabolism were consistent with the phenomena observed in in vivo hemolysis experiments.

\section{Lipidomic changes induced by OPD and OPD' in vivo}

A total of 82 lipids were identified as differential lipids. In each comparison group, the number of differential metabolites we found were 37 ( $\mathrm{NC}$ vs OPD), 31 (NC vs OPD') and 14 (OPD vs OPD'), respectively. As were shown in Table 4 and Fig. 5. Glycerophospholipids (GPs) and glycerolipids (GLs) was significantly perturbed. Among GPs, phosphatidylcholines (PC) changes accounted for the largest proportion, which was $48.65 \%$ (NC vs OPD), 54.05\% (NC vs OPD'), and 64.29\% (OPD vs OPD') respectively. This was consistent with the changing trend of different metabolites in metabolomics. Among GLs, compared with the NC group, when treated with OPD, there is a significant difference in the change of GLs compared with OPD'. However, when OPD compared with OPD', changes of GLs was similar.
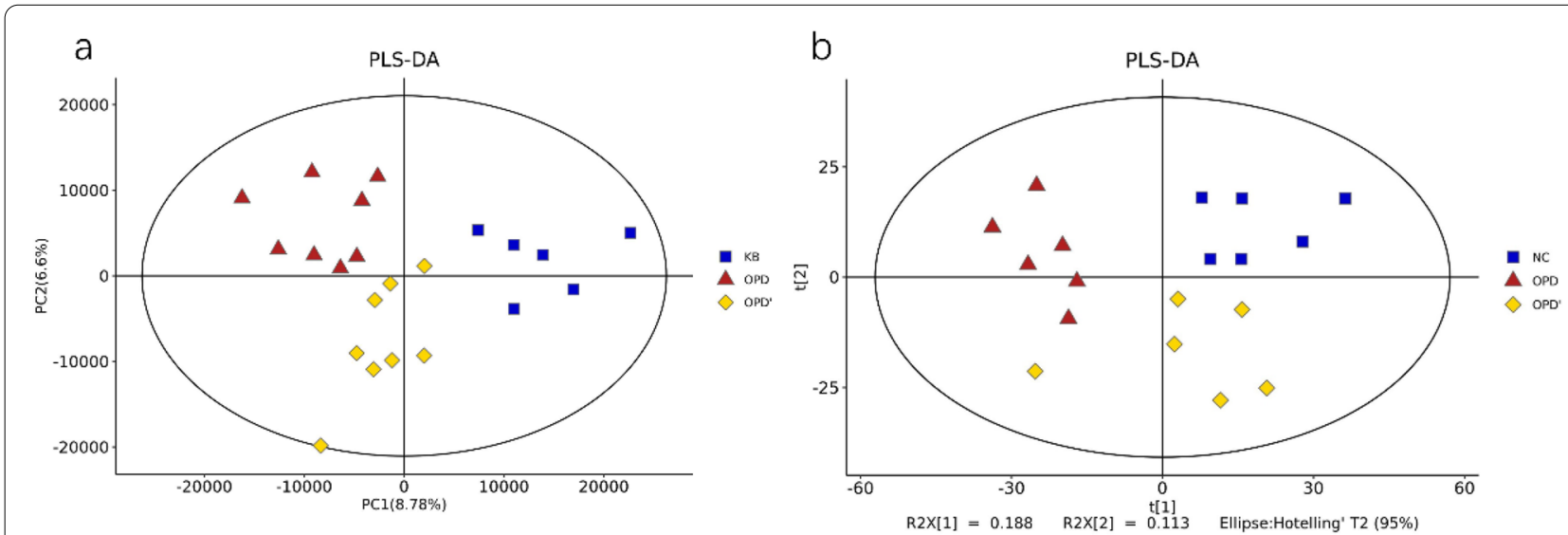

Fig. 3 Two-dimensional PLS-DA score plots of metabolic and lipidomic profiles in vivo with different drug treatment. a Metabolomic analysis in ESI positive and negative mode. $\mathbf{b}$ Lipidomic analysis in ESI positive and negative mode 
Table 3 Detailed information of top $20^{a}$ perturbed metabolites among different comparison group

\begin{tabular}{|c|c|c|c|c|c|c|c|c|c|}
\hline \multirow[t]{2}{*}{ Metabolites } & \multirow[t]{2}{*}{$\mathrm{m} / \mathrm{z}$} & \multirow[t]{2}{*}{$\begin{array}{l}\text { Retention } \\
\text { time (min) }\end{array}$} & \multirow[t]{2}{*}{ Detection mode } & \multicolumn{2}{|c|}{ (NC vs OPD) } & \multicolumn{2}{|c|}{$\begin{array}{l}\text { (NC vs } \\
\text { OPD') }\end{array}$} & \multicolumn{2}{|c|}{$\begin{array}{l}\text { (OPD vs } \\
\text { OPD') }^{\prime}\end{array}$} \\
\hline & & & & VIP & FC & VIP & FC & VIP & FC \\
\hline LysoPC $(18: 1(9 Z))^{b}$ & 522.3554 & 10.4072 & Positive & 40.29 & 1.23 & 42.27 & 1.22 & / & / \\
\hline$P C(16: 0 / 0: 0)[U] / P C(16: 0 / 0: 0)^{b}$ & 496.3396 & 9.8993 & Positive & / & / & 22.25 & 1.13 & / & / \\
\hline Cholic acid ${ }^{b}$ & 815.5688 & 8.2063 & Negative & / & / & / & / & 28.13 & 1.72 \\
\hline (R)-Butaprost & 834.6078 & 8.2002 & Positive & / & / & / & / & 20.9 & 1.55 \\
\hline PC $(14: 0 / 0: 0)^{b}$ & 468.3084 & 9.0744 & Positive & 17.68 & 1.95 & 13.98 & 1.48 & 6.50 & 0.76 \\
\hline LysoPC (16:1 (9z)/0:0) & 538.3154 & 9.3950 & Negative & 14.43 & 1.92 & 11.36 & 1.41 & 7.53 & 0.74 \\
\hline LysoPC $(20: 2(11 Z, 14 Z))^{b}$ & 548.3709 & 10.6952 & Positive & 14.12 & 1.30 & 16.65 & 1.34 & / & / \\
\hline LysoPC (20:1(11Z) $)^{b}$ & 550.3865 & 11.3843 & Positive & 12.81 & 1.22 & 16.56 & 1.32 & / & / \\
\hline 1-heptadecanoyl-sn-glycero-3-phosphocholine ${ }^{b}$ & 510.3555 & 10.6233 & Positive & / & / & 15.9 & 1.15 & / & / \\
\hline LysoPC $(16: 0)^{b}$ & 540.3312 & 9.8953 & Negative & / & / & 11.4 & 1.14 & / & / \\
\hline 3alpha-Hydroxy-5beta-chola-7,9(11)-dien-24-oic Acid & 373.2735 & 8.2002 & Positive & 12.72 & 0.73 & / & / & 20.47 & 1.53 \\
\hline LysoPC $(22: 5(7 Z, 10 Z, 13 Z, 16 Z, 19 Z))^{b}$ & 570.3551 & 10.0146 & Positive & 12.70 & 1.36 & 8.09 & 1.29 & / & / \\
\hline 15-hydroxy-tetracosa-6,9,12,16,18-pentaenoic acid & 357.2785 & 9.3971 & Positive & 11.09 & 0.56 & / & / & / & / \\
\hline Sulfolithocholylglycine & 512.2690 & 6.0969 & Negative & / & / & / & / & 9.80 & 1.74 \\
\hline LysoPC $(20: 3(5 Z, 8 Z, 11 Z))^{b}$ & 590.3467 & 10.1463 & Negative & 10.17 & 1.46 & 8.86 & 1.3 & / & / \\
\hline PC $(17: 1(10 Z) / 0: 0)^{b}$ & 508.3393 & 9.8993 & Positive & 9.18 & 1.67 & 7.65 & 1.37 & 3.54 & 0.82 \\
\hline (22E)-3beta-Hydroxy-5alpha-chola-7,22-dien-24-oic Acid & 373.2735 & 7.4742 & Positive & 9.18 & 0.57 & / & / & 14.6 & 2.12 \\
\hline LysoPC $(14: 0 / 0: 0)^{b}$ & 512.2997 & 9.0829 & Negative & 8.99 & 1.99 & 6.92 & 1.47 & 4.15 & 0.74 \\
\hline$P C(19: 1(9 Z) / 0: 0)^{b}$ & 536.3708 & 10.8693 & Positive & 8.90 & 1.61 & 8.55 & 1.51 & / & / \\
\hline Isocitrate & 191.0189 & 0.7660 & Negative & 8.35 & 1.74 & / & / & / & / \\
\hline$P C(0-18: 1(11 Z) / 0: 0)^{b}$ & 508.3762 & 10.7094 & Positive & 8.22 & 0.79 & / & / & / & / \\
\hline LysoPC $(17: 0)^{b}$ & 554.3466 & 10.6250 & Negative & 7.90 & 1.14 & 10.33 & 1.21 & / & / \\
\hline 3alpha,7alpha,12alpha-Trihydroxy-5alpha-cholan-24-al & 783.5790 & 9.3950 & Negative & 7.50 & 0.50 & / & / & / & / \\
\hline 2-Hydroxycinnamic acid & 182.0810 & 1.1162 & Positive & 6.38 & 1.16 & 8.68 & 1.22 & / & / \\
\hline Avicholic acid & 391.2840 & 7.6371 & Positive & 5.47 & 0.58 & / & / & 3.91 & 1.42 \\
\hline LysoPC $(20: 5(5 Z, 8 Z, 11 Z, 14 Z, 17 Z))^{b}$ & 586.3156 & 9.2737 & Negative & 4.98 & 1.41 & / & / & 4.66 & 0.78 \\
\hline 3alpha,9alpha, 11 beta-Trihydroxy-5beta-cholan-24-oic Acid & 391.2840 & 7.4742 & Positive & / & / & / & / & 5.62 & 2.17 \\
\hline$P C(16: 0 / 18: 2(9 Z, 12 Z))^{b}$ & 780.5518 & 13.6416 & Positive & / & / & 6.42 & 0.68 & / & / \\
\hline 4-methoxy-1-benzofuran-6-ol & 182.0811 & 0.7787 & Positive & / & / & 4.76 & 1.42 & / & / \\
\hline Scyphostatin A & 512.3348 & 9.2759 & Positive & / & / & 4.68 & 1.48 & / & / \\
\hline $\mathrm{p}$-Cresol sulfate & 187.0062 & 4.5210 & Negative & / & / & 4.37 & 0.31 & / & / \\
\hline L-Carnitine & 162.1123 & 0.7510 & Positive & / & / & 4.16 & 0.75 & / & / \\
\hline 2-Hydroxy-3-methoxyestrone & 424.3418 & 9.6654 & Positive & / & / & / & / & 4.68 & 1.36 \\
\hline LysoPC $(P-18: 0)^{b}$ & 552.3678 & 10.7062 & Negative & / & / & / & / & 4.48 & 1.22 \\
\hline Diosgenin & 397.3101 & 9.9280 & Positive & / & / & / & / & 3.81 & 0.56 \\
\hline 21-Deoxycortisol & 347.2216 & 7.2521 & Positive & / & / & / & / & 3.60 & 1.19 \\
\hline 12-Hydroxy-12-octadecanoylcarnitine & 426.3573 & 10.1025 & Positive & / & / & / & / & 3.44 & 1.40 \\
\hline 3alpha,12beta-Dihydroxy-11-oxo-5beta-cholan-24-oic Acid & 424.3056 & 8.1553 & Positive & / & / & / & / & 3.19 & 1.74 \\
\hline Americine & 584.2627 & 13.9012 & Positive & / & / & / & / & 2.97 & 1.89 \\
\hline LysoPE(20:4(8Z,11Z,14Z,17Z)/0:0) ${ }^{\text {b }}$ & 502.2926 & 9.7375 & Positive & / & / & / & / & 2.92 & 1.23 \\
\hline
\end{tabular}

"a" The ranking of top 20 is in descending order of VIP value. "b"Means that the metabolites were correlated to hemolysis

In the three comparison groups, there was another pattern that makes people confused. Lysophosphatidylcholine (LPC), the main component that causes hemolysis, has been down-regulated when treated with OPD and OPD', this was not consistent with the observed hemolytic behavior. 


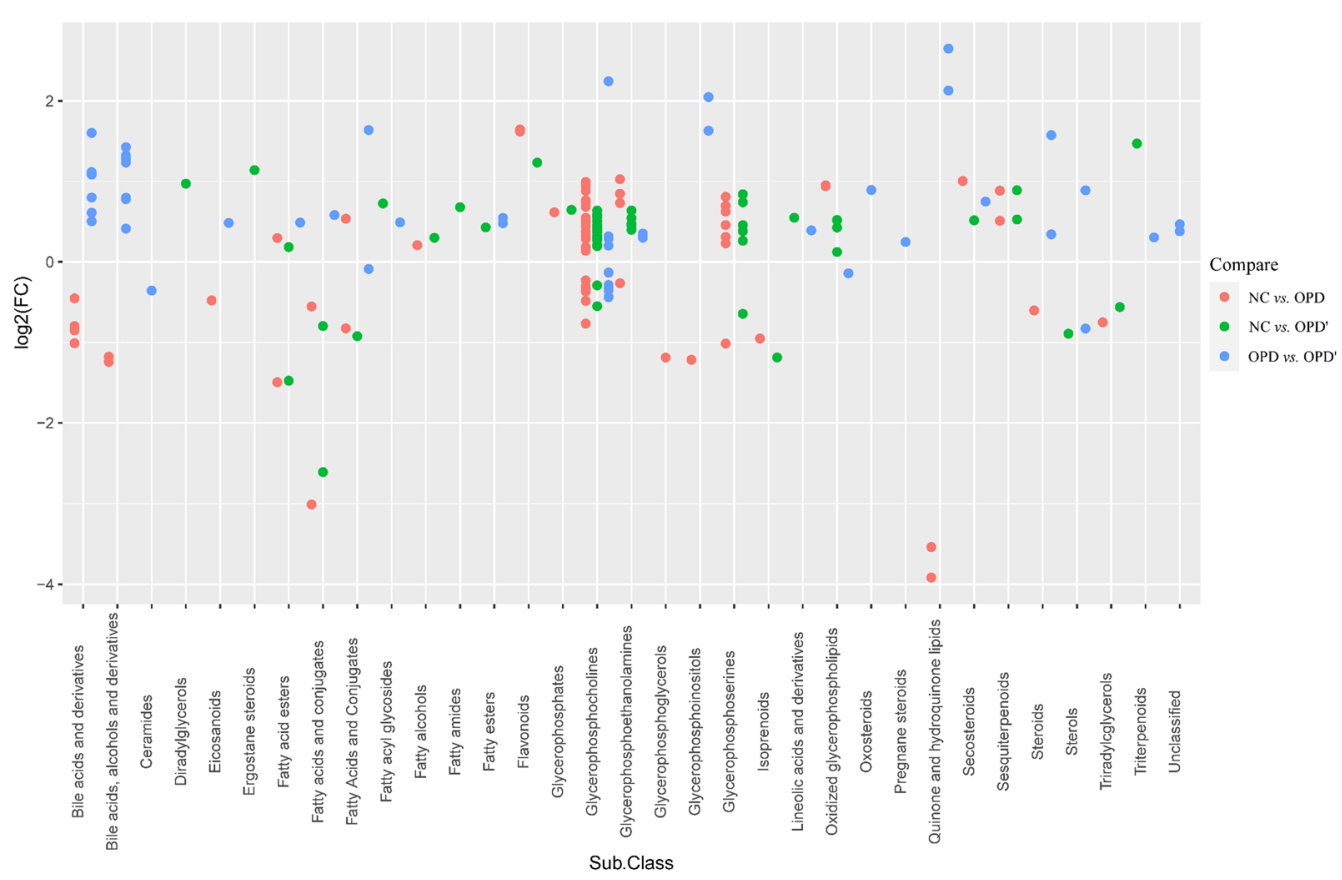

Fig. 4 The fold changes of differential metabolites among each comparison group from the perspective of sub class

\section{Metabolic and Lipidomic pathway analysis}

In order to further explore the hemolysis mechanism of OPD and OPD', the different metabolites in the plasma of rats in the OPD and OPD' group were substituted into the progqenesis QI data processing platform for metabolic path analysis, as shown in Fig. 6. The results showed that the top-20 metabolic pathways disturbed by OPD or OPD' were similar. Since the pathway of lipid metabolites is insufficient in the KEGG website, not all differential metabolites can be reflected in KEGG pathway among the screened differential metabolites, this is also a challenge for current pathway analysis. However, the trend of the Fig. 6 is consistent with the results of the differential metabolites which were shown in Table 3 , its predictions were still very informative.

Compared with NC group, choline metabolism in cancer and glycerophospholipid metabolism pathways were the most significantly enriched and largest number of differential metabolites gathered pathways in OPD and OPD' group, from a super class perspective, these two metabolic pathways were related to lipids and lipid-like molecules. As we all know, the main body of the cell membrane is the phospholipid bilayer and the fundamental cause of hemolysis of red blood cells is that the membrane was damaged. Therefore, we can speculate that the hemolysis caused by OPD and OPD' in vivo was related to the interference of glycerophospholipid metabolism. In addition, the pathways that were significantly affected also include pyrimidine metabolism, biosynthesis of amino acids, phenylalanine metabolism and bile secretion, these pathways not only have a certain relationship with the metabolism of glycerophospholipids, but may also participate in the metabolism and activation of OPD in vivo, which may result in the occurrence of OPD hemolysis.

\section{Discussion}

From the perspective of the chemical structure and physical and chemical properties of OPD and OPD', hemolysis caused by OPD' can be reasonably explained by its chemical structure, but OPD cannot [24-26]. In another article we published [27], relationship of hemolytic behavior in vitro of OPD and OPD' was studied. The results showed that OPD' has hemolytic effect in vitro, but OPD does not. In other words, OPD' hemolysis occurred both in vivo and in vitro, but the OPD only occurred in vivo. This indicates that the structure of OPD' is more likely to cause hemolysis. Meanwhile, there is another possible explanation for this phenomenon, the one is that OPD is bio-transformed into OPD' or its analogues in vivo, the other one is that both OPD and OPD' were metabolized 
Table 4 Detailed information of perturbed glycerophospholipids among different comparison group

\begin{tabular}{|c|c|c|c|c|c|c|c|c|c|}
\hline \multirow[t]{2}{*}{ Metabolites } & \multirow[t]{2}{*}{$\mathrm{m} / \mathrm{z}$} & \multirow{2}{*}{$\begin{array}{l}\text { Retention time } \\
\text { (min) }\end{array}$} & \multirow[t]{2}{*}{ Detection mode } & \multicolumn{2}{|c|}{ (NC vs. OPD) } & \multicolumn{2}{|c|}{ (NC vs. OPD') } & \multicolumn{2}{|c|}{ (OPD vs. OPD') } \\
\hline & & & & VIP & FC & VIP & $\mathrm{FC}$ & VIP & $\mathrm{FC}$ \\
\hline $\operatorname{LPC}(16: 1)$ & 538.3150 & 2.1049 & Negative & 1.80 & 0.54 & 1.69 & 0.68 & / & / \\
\hline MePC(19:4e) & 566.3217 & 2.3768 & Positive & 1.94 & 1.50 & / & / & 1.51 & 0.82 \\
\hline MePC(38:8e) & 824.5565 & 9.3435 & Positive & 1.32 & 1.72 & 1.23 & 1.45 & / & / \\
\hline PC(16:1_18:2) & 800.5447 & 6.9216 & Negative & 1.46 & 0.57 & / & / & / & / \\
\hline PC(18:1_22:6) & 876.5760 & 7.5938 & Negative & 1.42 & 0.58 & 1.38 & 0.67 & / & / \\
\hline PC(16:0_16:1) & 776.5447 & 8.1717 & Negative & 1.05 & 0.63 & / & / & 1.54 & 1.76 \\
\hline PC(40:6) & 834.6007 & 8.3262 & Positive & 1.13 & 0.71 & / & / & / & / \\
\hline PC(18:1_20:4) & 852.5760 & 8.1460 & Negative & 2.86 & 0.75 & / & / & 2.60 & 1.31 \\
\hline$P C(36: 2)$ & 786.6007 & 9.3590 & Positive & 8.17 & 1.24 & 6.62 & 1.12 & / & / \\
\hline$P C\left(18: 1 e \_16: 0\right)$ & 790.5967 & 9.6227 & Negative & 1.08 & 1.27 & / & / & / & / \\
\hline PC(18:1_18:1) & 808.5827 & 9.3608 & Positive & 2.70 & 1.35 & / & / & / & / \\
\hline PC(18:0_20:5) & 808.5851 & 9.3512 & Positive & 2.70 & 1.35 & / & / & / & / \\
\hline$P C(34: 1 e)$ & 746.6058 & 9.5947 & Positive & 1.07 & 1.39 & 1.11 & 1.28 & / & / \\
\hline PC(34:2e) & 744.5902 & 9.0343 & Positive & 1.03 & 2.37 & / & / & 1.07 & 0.47 \\
\hline PI(19:0e) & 613.3358 & 2.2930 & Negative & 1.02 & 0.79 & 1.54 & 0.73 & / & / \\
\hline $\operatorname{PS}(37: 2)$ & 800.5447 & 6.9216 & Negative & 1.46 & 0.57 & / & / & / & / \\
\hline $\mathrm{SPH}(\mathrm{d} 20: 0)$ & 330.3367 & 2.9837 & Positive & 1.50 & 1.56 & / & / & / & / \\
\hline SPH(t16:0) & 290.2690 & 1.2942 & Positive & 1.52 & 3.07 & 1.23 & 2.16 & / & / \\
\hline $\mathrm{CPA}(18: 2)$ & 415.2255 & 2.5302 & Negative & / & / & 1.08 & 0.40 & / & / \\
\hline$C P A(16: 0)$ & 391.2255 & 2.9310 & Negative & / & / & 1.41 & 0.53 & / & / \\
\hline CPA(18:0) & 419.2568 & 3.4579 & Negative & / & / & 1.16 & 0.57 & / & / \\
\hline $\mathrm{dMePE}(40: 7)$ & 816.5549 & 7.8982 & Negative & / & / & 2.49 & 1.83 & / & / \\
\hline $\operatorname{LPC}(17: 0)$ & 554.3463 & 3.0918 & Negative & / & / & 1.57 & 0.69 & / & / \\
\hline LPC(18:2) & 564.3307 & 2.4355 & Negative & / & / & 6.31 & 0.85 & / & / \\
\hline PC(20:4_20:4) & 874.5604 & 6.4729 & Negative & / & / & 1.26 & 0.77 & / & / \\
\hline PC(18:0_18:2) & 830.5917 & 9.3742 & Negative & / & / & 6.21 & 1.12 & / & / \\
\hline PC(16:0_18:1) & 804.5760 & 9.2239 & Negative & / & / & 4.46 & 1.15 & 5.42 & 1.28 \\
\hline$P C(34: 0)$ & 762.6007 & 9.8127 & Positive & / & / & 1.22 & 1.16 & / & / \\
\hline PC(18:1e_20:4) & 838.5967 & 8.9016 & Negative & / & / & 1.26 & 1.25 & / & / \\
\hline$P C\left(18: 1 e \_16: 0\right)$ & 790.5967 & 9.6227 & Negative & / & / & 1.32 & 1.26 & / & / \\
\hline PC(16:1e_20:4) & 810.5654 & 8.6947 & Negative & / & / & 1.13 & 1.79 & / & / \\
\hline LPC(18:0) & 568.3620 & 3.3742 & Negative & / & / & / & / & 3.49 & 0.84 \\
\hline$P C(34: 1)$ & 760.5851 & 9.2172 & Positive & / & / & / & / & 4.81 & 1.19 \\
\hline PC(16:0_16:0) & 778.5604 & 9.2035 & Negative & / & / & / & / & 2.45 & 1.29 \\
\hline PC(14:0_18:2) & 774.5291 & 6.7773 & Negative & / & / & / & / & 1.07 & 2.03 \\
\hline
\end{tabular}

into more activated forms for hemolysis. The inconsistency of OPD's hemolytic behavior in vivo and in vitro may be closely related to the metabolic process of OPD after administration.

When OPD was compared with OPD', the same differential metabolites based on comparison with $\mathrm{NC}$ group are neutralized, therefore, more details between OPD and OPD' in vivo metabolic processes were discovered.
In its top 20 differential metabolites, LysoPC account for $20 \%$, while cholic acid ranked first place (VIP value was 28.13 , Fold change value was 1.72). In another article we submitted, molecular modeling was adopted and screen out a protein called Q9NPD5 which can interact with both OPD and OPD'. This protein belongs to organic anion transporters (OATP) family and it was closely related to cholic acid metabolism [28], existing studies 


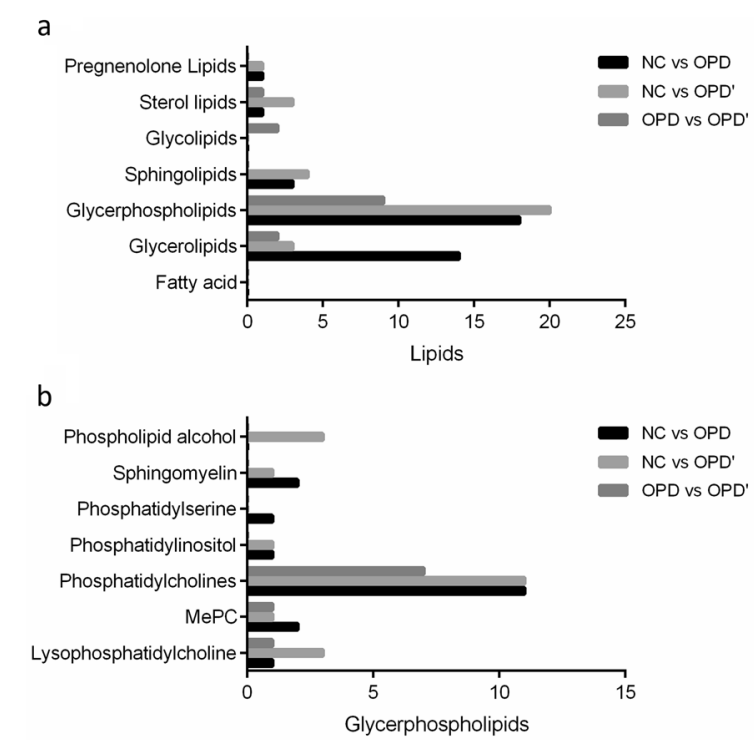

Fig. 5 The distribution of different types of differential lipids in each comparison group. a Based on the differential lipids. $\mathbf{b}$ Based on the glycerphospholipids

have confirmed that the down-regulation of OATP1A2 and OATP1B3 in particular leads to abnormal fetal bile acid metabolism between maternal and fetal fetuses [29]. This may explain from another perspective how OPD and OPD' induce hemolysis. OATP1B3, which is highly expressed in the liver, is localized at the plasma membrane at the subcellular level. When OPD or OPD' enters the liver for metabolism, it binds to OATP1B3 [30], which disturbs the fluidity of the cell membrane and further induces hemolysis.

Published literature [31] shows that OPD can regulate the metabolism of glucose and lipids and improve the metabolic syndrome by interfering with the types and proportions of gut microbiota when taken orally. However, oral administration is very different from intravenous administration. Judging from the changes in the metabolic and lipid profile of different groups, the disorder of phospholipid metabolism dominates the causes of OPD and OPD' induced hemolysis [32, 33]. Statistics of all differential metabolites from two levels of super class and class were used to analyze the proportion of lipids and glycerophospholipids in the metabolic profile. In the NC vs OPD group, the ratio of super class level, i.e. lipids and lipid-like molecules, to all differential metabolites was $67 / 145$, the ratio of class level, i.e. glycerophospholipids, to super class level's metabolites was $42 / 67$. In the other two groups, these two ratios were 60/124 (to all differential metabolites), 39/60 (to super class level's metabolites) in NC vs OPD' group and 52/84 (to all differential metabolites), 10/52 (to super class level's metabolites) in OPD vs OPD' group. Further analysis of sub-class metabolites at the class level, we found that at the sub-class level, the proportion of LysoPC was $18 / 42,13 / 39$ and $7 / 10$ respectively. These ratios have significant statistical differences in comparison with other categories of differential metabolites in their corresponding levels. The metabolism of glycerophospholipid to lysophospholipid requires two steps of catalysis by phospholipase A2 and phospholipase B2, and the resulting lysophospholipid contains a hydrophobic hydrocarbon chain and a polar phosphate group. It was a very strong surfactant and has strong ability to destroy cell membranes [34]. But how OPD and OPD' participated in this process remains to be further explored.

Our further analysis of differential metabolites shows that it is very difficult to clearly point out a specific metabolite or pathway. The results of metabolomics and lipidomics experiments show that the changes of differential metabolites have obvious enrichment characteristics, and they are all enriched in the metabolism of phospholipids, including LysoPC, PC and cholic acid et.al., the relationship between these differential metabolites and hemolysis is very close. However, the lack of enrichment pathways related to these differential metabolites (lipids and lipid related metabolites) hinders the analysis of related pathways and the further work.

\section{Conclusion}

UPLC-QE/MS was successfully applied to investigate the significant changes in plasma between OPD- and OPD'-treated rats. Both OPD and OPD' groups experienced hemolysis, subsequently, mechanism of differences in endogenous metabolites and lipids were investigated. Changes in endogenous differential metabolites and differential lipids, enrichment of differential metabolic pathways, and correlation analysis of differential metabolites and lipids all indicated that the causes of hemolysis by OPD and OPD' were closely related to the interference of phospholipid metabolism. This study provided a comprehensive description of metabolome and lipidomic changes between OPD- and OPD'-treated rats. However, the precise mechanism requires further exploration. 


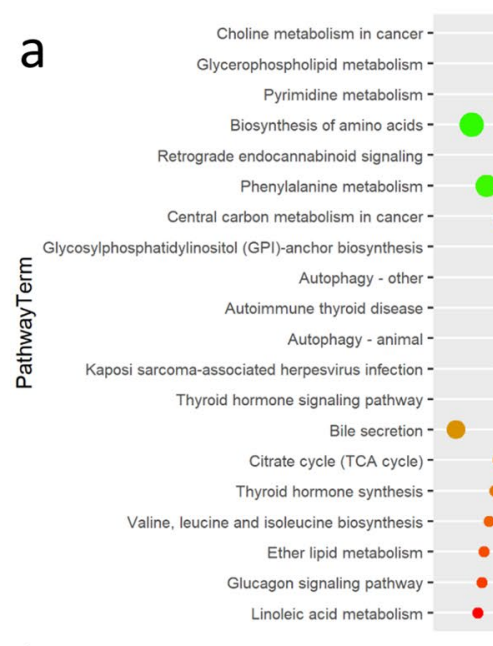

b
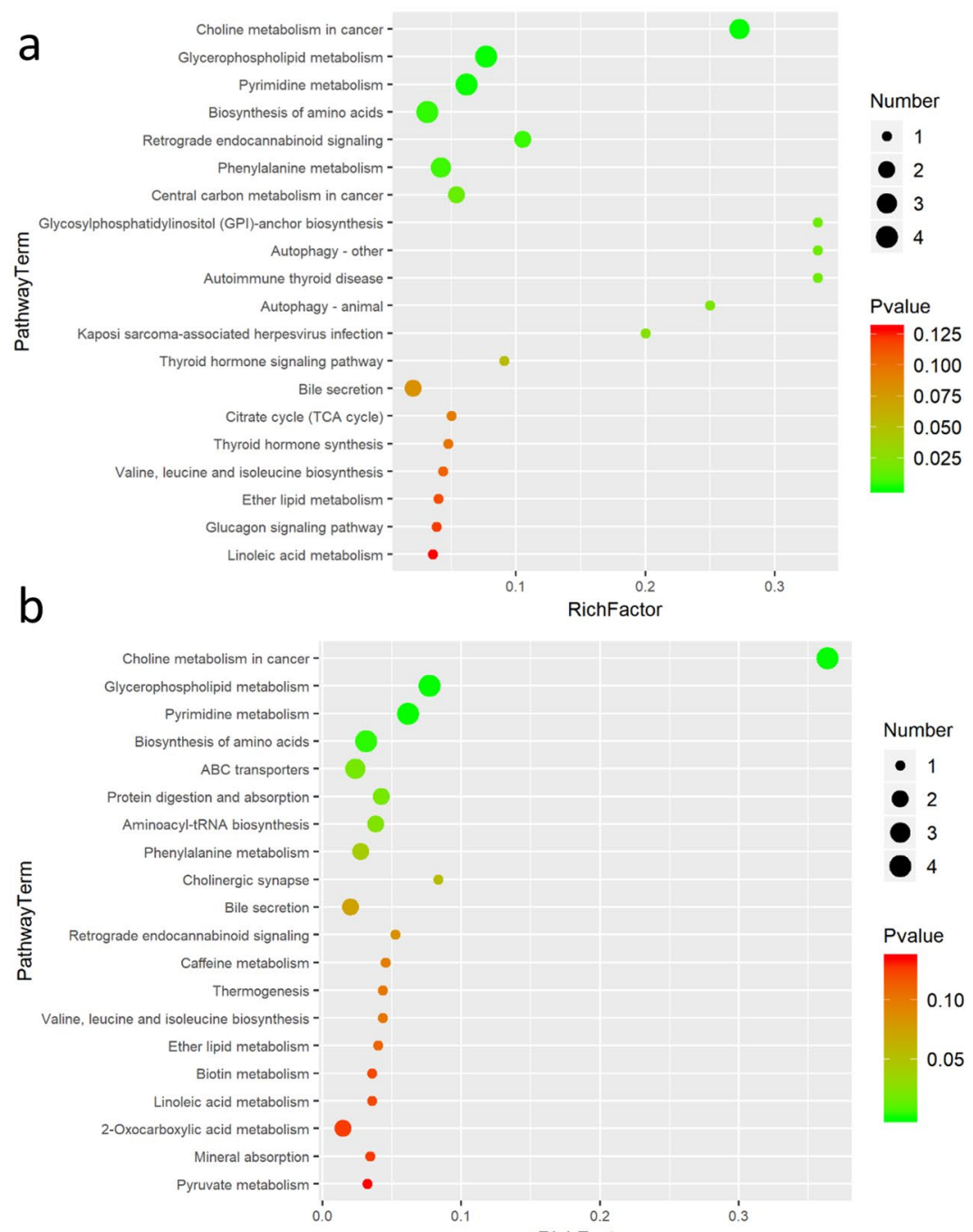

Number

- 1

- 2

3

4

Pvalue

0.10

0.05

C

RichFactor

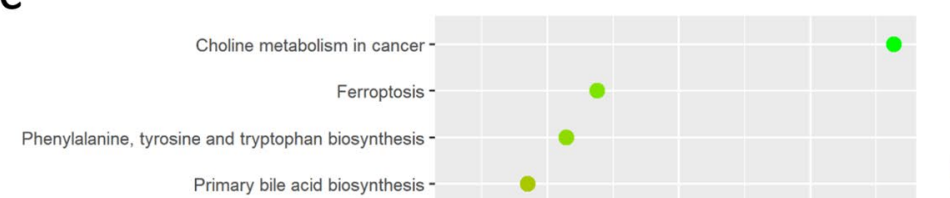

Number

- 1

竞

Glycerophospholipid metabolism -

Pvalue

Arginine and proline metabolism -

Purine metabolism -

Bile secretion -

Steroid hormone biosynthesis -

Biosynthesis of amino acids -

-

0.05

Fig. 6 Top-20 Disturbed metabolic pathways. a NC vs OPD group. b NC vs OPD' group. c OPD vs OPD'group 


\section{Supplementary Information}

The online version contains supplementary material available at https://doi. org/10.1186/s13020-020-00412-z.

Additional file 1: Figure S1. Volcano graph for screening differential metabolites. Figure $\mathbf{S 2}$. Volcano graph for screening differential lipids.

\section{Abbreviations}

OPD: Ophiopogonin D; OPD': Ophiopogonin D'; UPLC: Ultra-high performance liquid chromatography; PCA: Principal component analysis; OPLS-DA: Orthogonal partial least square-discriminant analysis; PLS-DA: Partial least square-discriminant analysis; VIP: Variable importance in the projection; SMI: Shenmai injection; TCM: Traditional Chinese medicine; ROS: Reactive oxygen species; HGB: Hemoglobin; RBC: Red blood cell; RET: Reticulocyte; LysoPC: Lysophospholipids; MS: Mass spectrometry; HESI: Heated electrospray ionization; URO: Urobilinogen; OATP: Organic anion transporters; GPs: Glycerophospholipids; GLs: Glycerolipids; LPC: Lysophosphatidylcholine.

\section{Acknowledgements}

The authors would like to thank Xiang-Tao Luo for supporting animal experiments.

\section{Authors' contributions}

H-HX, C-SH and Y-TS performed the experimental sections. L-LX support for the UPLC-MS/MS analysis. Z-HJ performed data analysis and visualization. $\mathrm{H}-\mathrm{HX}$ wrote the manuscript. All authors read and approved the final manuscript.

\section{Funding}

This research was funded by the National Natural Science Foundation of China (81703669) and National Science and Technology Major Project of "Major New Drug Creation" (2017ZX09301012-007).

\section{Availability of data and materials}

All data used to support the findings of this study are available from the corresponding author upon request.

\section{Ethics approval and consent to participate}

The experimental protocols were approved by the Animal Ethics Committee of Academy of Military Medical Sciences (No. IACUC-DWZX-2020-684).

\section{Consent for publication}

Not applicable.

\section{Competing interests}

The authors declare that they have no competing interests.

\section{Author details}

${ }^{1}$ Tianjin University of Traditional Chinese Medicine, Tianjin 300193, China 2 Department of Pharmaceutical Sciences, Beijing Institute of Radiation Medicine, Beijing 100850, China. ${ }^{3}$ Jiangxi Province Key Laboratory of Molecular Medicine, Nanchang 330006, China. ${ }^{4}$ Guangdong Pharmaceutical University, Guangzhou 510006, China. ${ }^{5}$ College of Life Science and Bioengineering, Beijing University of Technology, Beijing 100124, China.

Received: 13 August 2020 Accepted: 11 December 2020 Published online: 06 January 2021

\section{References}

1. Zhang S, You ZQ, Yang L, Li LL, Wu YP, Gu LQ, Xin YF. Protective effect of Shenmai injection on doxorubicin-induced cardiotoxicity via regulation of inflammatory mediators. BMC Complement Altern Med. 2019:19(1):317.

2. Li L, Li J, Wang Q, Zhao X, Yang D, Niu L, Yang Y, Zheng X, Hu L, Li Y. Shenmai injection protects against doxorubicin-induced cardiotoxicity via maintaining mitochondrial homeostasis. Front Pharmacol. 2020;11:815.
3. Zhang XY, Sun Y, Yang XY, Hu JY, Zheng R, Chen SQ, Li M, Li CY, Jiang Y, Liu $S$, et al. Effect of Chinese medicine on no or slow reflow after percutaneous coronary intervention in myocardial infarction patients: a systematic review and meta-analysis. Chin J Integr Med. 2020;26(3):227-34.

4. Xian S, Yang Z, Lee J, Jiang Z, Ye X, Luo L, Jin L, Yang T, Ye S, Lu D. A randomized, double-blind, multicenter, placebo-controlled clinical study on the efficacy and safety of Shenmai injection in patients with chronic heart failure. J Ethnopharmacol. 2016;186:136-42.

5. Zhang $C$, Zheng Y, Chen T, Wang S, Xu M. The utility of traditional Chinese medicine (Shenmai) in the cardiac rehabilitation after coronary artery bypass grafting: a single-center randomized clinical trial. Complement Ther Med. 2019;47:102203.

6. Liu ZL, Liu ZJ, Liu JP, Kwong JS: Herbal medicines for viral myocarditis. In: Cochrane Database of Systematic Reviews: Reviews, Chichester: John Wiley \& Sons, Ltd. https://doi.org/10.1002/14651858.CD003711.pub4.

7. Wang K, Wu J, Wang H, Duan X, Zhang D, Wang Y, Ni M, Liu S, Meng Z, Zeng $X$, et al. Comparative efficacy of chinese herbal injections for pulmonary heart disease: a bayesian network meta-analysis of randomized controlled trials. Front Pharmacol. 2020;11:634.

8. Zhou Q, Sun Y, Tan W, Liu X, Qian Y, Ma X, Wang T, Wang X, Gao X. Effect of Shenmai injection on preventing the development of nitroglycerininduced tolerance in rats. PLoS ONE. 2017:12(4):e0176777.

9. Zhou Y, Zhao B, Wu W, Yang X, Long S, Deng H, He W, Liao G, Li Q, Xie $Z$. Shenmai injection for the treatment of cancer-related fatigue in advanced non-small cell lung cancer patients undergoing chemotherapy: study protocol for a randomized controlled trial. Trials. 2018;19(1):474.

10. Lu LY, Zheng GQ. An overview of systematic reviews of shenmai injection for healthcare. Evid Based Complement Alternat Med. 2014;2014:840650.

11. Duan B, Xie J, Rui Q, Zhang W, Xi Z. Effects of Shengmai injection add-on therapy to chemotherapy in patients with non-small cell lung cancer: a meta-analysis. Support Care Cancer. 2018;26(7):2103-11.

12. [The epidemiological characteristics of an outbreak of 2019 novel coronavirus diseases (COVID-19) in China]. Zhonghua Liu Xing Bing Xue Za Zhi 2020, 41(2):145-151.

13. China TSCIOotPsRo: The State Council Office held a conference on the important role of Chinese medicine in the prevention and treatment of new coronary pneumonia and effective drugs. [EB/OL] 2020: http://www. scio.gov.cn/xwfbh/xwbfbh/wafbh/42311/42768/index.html. Accessed 23 Mar 2020.

14. Xiaoyan H, Yuguang W, Zhaoyan Z, Yuan W, Xiangmei C, Yi W, Yue G. Ophiopogonin $\mathrm{D}$ and EETs ameliorate Ang Il-induced inflammatory responses via activating PPARa in HUVECs. Biochem Biophys Res Commun. 2017. https://doi.org/10.1016/j.bbrc.2017.06.007.

15. Wen-Ting $Y$, Tao Z, Zeng-Chun M, Qian-de L, Cheng-Rong X, Xiang-Lin T, Hong-Ling T, Bo-Li Z, Yu-Guang W, Yue G. Ophiopogonin D maintains $\mathrm{Ca}(2+)$ homeostasis in rat cardiomyocytes in vitro by upregulating CYP2J3/EETs and suppressing ER stress. Acta Pharmacol Sin. 2016. https:// doi.org/10.1038/aps.2015.146.

16. Xiaoyan $H$, Yuguang W, Yi W, Liang Y, Jia W, Yue G. Ophiopogonin D reduces myocardial ischemia-reperfusion injury via upregulating CYP2J3/ EETs in rats. Cell Physiol Biochem. 2018. https://doi.org/10.1159/00049 3500.

17. Zongliang L, He W, Mingxing Z, Wei S, Jiajia W, Changpeng W, Ya K, Jing G, Na L, Jie L, et al. Ophiopogonin D', a natural product from radix ophiopogonis, induces in vitro and in vivo RIPK1-dependent and caspaseindependent apoptotic death in androgen-independent human prostate cancer cells. Front Pharmacol. 2018. https://doi.org/10.3389/fphar .2018.00432.

18. Zongliang L, Changpeng W, Mingxing Z, Wei S, He W, Jiajia W, Jing G, Na L, Jie L, Yanwu L, et al. Ophiopogonin D'induces RIPK1-dependent necroptosis in androgen-dependent LNCaP prostate cancer cells. Int J Oncol. 2020;56(2):439-47.

19. Si-Jia R, Huan-hua X, Ming L, Fei-ran H, Zeng-chun M, Xiang-lin T, Qiande L, Hong-ling T, Cheng-rong X, Yu-guang W, et al. Cytotoxicity of ophiopogonin D'for rat H9c2 cardiomyocytes. Chin J Pharmacol Toxicol. 2017:31(04):325-31.

20. Wang J, Wang NN, Ge YX, Tan HL, Ma ZC, Wang YG, Gao Y. Ophiopogonin $D$ protects cardiomyocytes against ophiopogonin $D^{\prime}$-induced injury through suppressing endoplasmic reticulum stress. Zhongguo Zhong Yao Za Zhi. 2019;44(9):1876-81. 
21. Sheng-cai G. Literature analysis of adverse reactions induced by Shenmai injection. Chin J Drug App Monitor. 2014;11(02):115-7.

22. Sears DA, Anderson PR, Foy AL, Williams HL, Crosby WH. Urinary iron excretion and renal metabolism of hemoglobin in hemolytic diseases. Blood. 1966;28(5):708-25

23. Merle NS, Grunenwald A, Rajaratnam H, Gnemmi V, Frimat M, Figueres ML, Knockaert S, Bouzekri S, Charue D, Noe R, et al. Intravascular hemolysis activates complement via cell-free heme and heme-loaded microvesicles. JCI Insight. 2018. https://doi.org/10.1172/jci.insight.96910.

24. Seeman $P$, Cheng D, lles GH. Structure of membrane holes in osmotic and saponin hemolysis. J Cell Biol. 1973;56(2):519-27.

25. Manaargadoo-Catin M, Ali-Cherif A, Pougnas JL, Perrin C. Hemolysis by surfactants-A review. Adv Colloid Interface Sci. 2016;228:1-16.

26. Chen Z, Duan H, Tong X, Hsu P, Han L, Morris-Natschke SL, Yang S, Liu W, Lee KH. Cytotoxicity hemolytic toxicity, and mechanism of action of pulsatilla saponin D and its synthetic derivatives. J Nat Prod. 2018;81(3):465-74.

27. Hong Y, Jianqiu L, Huanhua X, Liang Y, Yao N, Yuhao N, La J, Xianglin T, Zengchun M, Yuguang W, et al. Established UPLC-MS / MS method to determinate ophiopogonin D and ophiopogonin D' and clinical risk assessment. Drug Eval Res. 2019;42(06):1135-40.

28. Liu X, Chen L, Liu M, Zhang H, Huang S, Xiong Y, Xia C. Ginsenoside $\mathrm{Rb} 1$ and $\mathrm{Rd}$ remarkably inhibited the hepatic uptake of Ophiopogonin $\mathrm{D}$ in Shenmai injection mediated by OATPs/oatps. Front Pharmacol. 2018;9:957.

29. Zhang W, Xiong X, Chen L, Liu M, Xiong Y, Zhang H, Huang S, Xia C. Hepatic uptake mechanism of Ophiopogonin d mediated by organic anion transporting polypeptides. Eur J Drug Metab Pharmacokinet. 2017:42(4):669-76.

30. Chen L, Liu L, Chen Y, Liu M, Xiong Y, Zhang H, Huang S, Xia C. Modulation of transporter activity of OATP1B1 and OATP1B3 by the major active components of Radix Ophiopogonis. Xenobiotica. 2019;49(10):1221-8.

31. Siyu C, Xiao L, Li L, Chang L, Xiao H. Ophiopogonin D alleviates high-fat diet-induced metabolic syndrome and changes the structure of gut microbiota in mice. FASEB Journal. 2018. https://doi.org/10.1096/f.20170 0741RR.

32. Ochi S, Hashimoto K, Nagahama M, Sakurai J. Phospholipid metabolism induced by Clostridium perfringens alpha-toxin elicits a hot-cold type of hemolysis in rabbit erythrocytes. Infect Immun. 1996;64(9):3930-3.

33. Chaves-Moreira D, Souza FN, Fogaça RT, Mangili OC, Gremski W, SenffRibeiro A, Chaim OM, Veiga SS. The relationship between calcium and the metabolism of plasma membrane phospholipids in hemolysis induced by brown spider venom phospholipase-D toxin. J Cell Biochem. 2011;112(9):2529-40.

34. Behling-Kelly EL, Wakshlag J. A commercial soy-based phospholipid emulsion accelerates clot formation in normal canine whole blood and induces hemolysis in whole blood from normal and dogs with inflammatory leukograms. J Vet Emerg Crit Care (San Antonio). 2018;28(3):252-60.

\section{Publisher's Note}

Springer Nature remains neutral with regard to jurisdictional claims in published maps and institutional affiliations.
Ready to submit your research? Choose BMC and benefit from:

- fast, convenient online submission

- thorough peer review by experienced researchers in your field

- rapid publication on acceptance

- support for research data, including large and complex data types

- gold Open Access which fosters wider collaboration and increased citations

- maximum visibility for your research: over $100 \mathrm{M}$ website views per year

At BMC, research is always in progress.

Learn more biomedcentral.com/submissions 